\title{
THE SPEED OF EXTINCTION FOR SOME GENERALIZED JIŘINA PROCESSES
}

\author{
YUQIANG LI,* East China Normal University
}

\begin{abstract}
The speed of extinction for some generalized Jiřina processes $\left\{X_{n}\right\}$ is discussed. We first discuss the geometric speed. Under some mild conditions, the results reveal that the sequence $\left\{c^{n}\right\}$, where $c$ does not equal the pseudo-drift parameter at $x=0$, cannot estimate the speed of extinction accurately. Then the general case is studied. We determine a group of sufficient conditions such that $X_{n} / c_{n}$, with a suitable constant $c_{n}$, converges almost surely as $n \rightarrow \infty$ to a proper, nondegenerate random variable. The main tools used in this paper are exponent martingales and stochastic growth models.
\end{abstract}

Keywords: Generalized Jiřina process; extinction; geometric series

2000 Mathematics Subject Classification: Primary 60J80

Secondary 60F15; 60J10

\section{Introduction}

The generalized Jiřina process considered in this paper is a modification of the Jirrina process [1] whose reproductive behavior may depend on the size of the population. This kind of process was first introduced by $\mathrm{Li}$ [4] and called the continuous-state population-size-dependent branching process (continuous-state PSDBP). Here we recall its definition.

A time-homogeneous Markov process $X=\left\{X_{n}\right\}$ with state space $[0, \infty)$ is called a continuous-state PSDBP if, for any $\lambda \geq 0$ and $x \geq 0$,

$$
\mathrm{E}\left[\exp \left\{-\lambda X_{n+1}\right\} \mid X_{n}=x\right]=\exp \{-x F(x, \lambda)\},
$$

where $F(x, \lambda)$ is a reproduction cumulative function (RCF) satisfying

$$
F(x, \lambda)=\gamma(x) \lambda+\int_{0+}^{+\infty}\left(1-\mathrm{e}^{-\lambda u}\right) v(x, \mathrm{~d} u) .
$$

Here $\gamma(x)$ is a nonnegative Borel function and $(1 \wedge u) v(x, \mathrm{~d} u)$ is a finite kernel from $[0,+\infty)$ to $(0,+\infty)$. Obviously, the continuous-state PSDBP is uniquely determined by the function $F(x, \lambda)$ or the functions $\gamma(x)$ and $v(x, \mathrm{~d} u)$. We call $\gamma(x)$ the drift function, $v(x, \cdot)$ the Lévy measure function, and

$$
m(x):=\gamma(x)+\int_{0+}^{+\infty} u v(x, \mathrm{~d} u)
$$

the offspring mean function if the integral is finite. Furthermore, we call the limit of $\gamma(x)$ as $x \rightarrow 0$ the pseudo-drift at $x=0$ and the limit of $m(x)$ as $x \rightarrow 0$ the pseudo-mean at $x=0$. In general, we call $X$ the subcritical continuous-state PSDBP if its offspring mean function

Received 19 September 2008; revision received 24 March 2009.

* Postal address: School of Finance and Statistics, East China Normal University, Shanghai 200241, P. R. China.

Email address: yqli@stat.ecnu.edu.cn 
$m(x)$ is bounded and $\lim _{x \rightarrow+\infty} m(x)<1$, or the supercritical continuous-state PSDBP if $\lim _{x \rightarrow+\infty} m(x)>1$.

Obviously, if $\gamma(x) \equiv \gamma$ is a constant function and $v(x, \mathrm{~d} s) \equiv v(\mathrm{~d} s)$ for some measure $v(\mathrm{~d} s)$ on $(0, \infty)$, then $X=\left\{X_{n}\right\}$ is a Jiřina process. Hence, for Jiřina processes, their offspring mean functions are constants.

It is easy to check that if $m(x)$ exists then, for any $k \geq 0$,

$$
\mathrm{E}\left[X_{k+1} \mid X_{k}\right]=m\left(X_{k}\right) X_{k} .
$$

Since continuous-state PSDBPs and Jiřina processes have a similar construction, it seems reasonable to imagine that they have similar properties, though there are some complexities that require a more comprehensive approach and lead to more difficulties in studying continuousstate PSDBPs. For example, a continuous-state PSDBP can arise from the limit of a sequence of scaled generalized Galton-Watson processes (see [4]), while a Jiřina process can arise from a sequence of scaled Galton-Watson processes. Under some mild conditions, the continuousstate PSDBPs have the extinct property

$$
\mathrm{P}\left(X_{n} \rightarrow 0 \text { or } X_{n} \rightarrow+\infty\right)=1,
$$

which is the same as that of the Jiřina processes, though we cannot use the composition of the RCF $F(x, \lambda)$ (see [7]). In addition, Li [5], [6] showed that, under certain conditions, a sequence of scaled continuous-state PSDBPs converges weakly to a diffusion process whose stochastic differential equation is similar to that of the limiting diffusion arising from a sequence of scaled Jiřina processes.

Note that Jiřina [2] raised the following question concerning Jiřina processes: if the Jiřina process $X=\left\{X_{n}\right\}$ is subcritical, so that in fact $X_{n} \rightarrow 0$ almost everywhere, do there exist positive constants $c_{n}$ such that $\left\{X_{n} / c_{n}\right\}$ approaches a nondegenerate random variable in some sense? Seneta and Vere-Jones [8] obtained a necessary and sufficient condition in the weak convergence sense, i.e. that $\gamma>0$ and $\left\{c_{n}\right\}$ satisfy $\lim _{n \rightarrow+\infty} c_{n+1} / c_{n}=\gamma$, where $\gamma$ is the drift constant of $X$. At the same time, Seneta and Vere-Jones pointed out that if $X$ is a supercritical Jiřina process then $X_{n} / c_{n}$ with $\lim _{n \rightarrow+\infty} c_{n+1} / c_{n}=m$ converges weakly to a proper, nondegenerate random variable, where $m$ is the offspring mean of $X$. We remark that Kesten pointed out in his report of [8] (see MR0246379 in Mathematical Reviews) that strong convergence can be readily obtained f rom weak convergence.

Natural questions to ask are what is the speed of extinction for the continuous-state PSDBPs and whether the results proved by Seneta and Vere-Jones [8] still hold to some extent for continuous-state PSDBPs? We note that the results in [8] show that in order to obtain a proper, nondegenerate limit for $X_{n} / c_{n},\left\{c_{n}\right\}$ must depend on $\gamma$ for the subcritical case and on $m$ for the supercritical case. It seems that the convergence mechanisms of $X_{n} / c_{n}$ are different for the subcritical case and the supercritical case. Is this difference essential? In this work we aim to provide some preliminary discussions of these problems. We will seek some sufficient conditions such that $X_{n} / c_{n}$ converges almost surely (a.s.) to a proper, nondegenerate random variable, where $X_{n}$ is a continuous-state PSDBP with $\lim _{n \rightarrow+\infty} X_{n}=0$ a.s. Our results to some extent generalize the corresponding results of [8]. Applying our results to Jiřina processes, we can define the parameter $1 / \gamma$ as the 'mean' in some sense; therefore, we can say in some sense that the results of [8] for the subcritical and supercritical cases are consistent. For more details, see Subsection 4.2, below.

The main tools used in this paper are exponent martingales and general stochastic growth models (see, for example, [3]). We divide this paper into four sections. The basic assumptions 
and the main results are introduced in Section 2. In Section 3 we investigate the possible geometric speed of extinction. In Section 4 we formulate a stochastic growth model based on the continuous-state PSDBP and use it to find a group of sufficient conditions such that $X_{n} / c_{n}$ converges almost everywhere to a proper, nondegenerate random variable.

\section{Basic assumptions and main results}

From now on, $X=\left\{X_{n}, n=0,1,2, \ldots\right\}$ is a continuous-state PSDBP with $X_{0}>0$ and $\mathrm{RCF}$

$$
F(x, \lambda)=\gamma(x) \lambda+\int_{0+}^{+\infty}\left(1-\mathrm{e}^{-\lambda s}\right) v(x, \mathrm{~d} s),
$$

where $1>\gamma(x)>0$. In addition, unless stated otherwise, we define

$$
F(\lambda)=\gamma \lambda+\int_{0+}^{+\infty}\left(1-\mathrm{e}^{-\lambda u}\right) \mu(\mathrm{d} u),
$$

where $0<\gamma<1$ and $\mu$ is a measure on $(0,+\infty)$ with $\int_{0+}^{+\infty}(1 \wedge u) \mu(\mathrm{d} u)<+\infty$.

Note that $X_{0}>0$ and $\gamma(\cdot)>0$ imply that, for any $n \geq 0, X_{n}>0$ a.s.

Throughout this paper, we suppose that $X$ becomes extinct, i.e. $X_{n} \rightarrow 0$ a.s. as $n \rightarrow+\infty$. According to [7], this is not an extraordinary assumption. If $F(x, \lambda)$ is continuous on $x \in$ $[0,+\infty)$ for any $\lambda>0$ and the criterion function

$$
f(x)=\sup \{\lambda \geq 0: \lambda \leq F(x, \lambda)\}
$$

is bounded, then the subcritical continuous-state PSDBP becomes extinct.

Let $\left\{c_{n}\right\}$ be a sequence of positive constants, and, for any $n \geq 0$, define

$$
Y_{n}=\frac{X_{n}}{c_{n}} .
$$

We first consider the special case in which $c_{n}=c^{n}$ for some $c \in(0,1)$. To this end, we assume that

(A) as $x \rightarrow 0, \gamma(x) \rightarrow \gamma$ and $F(x, \lambda) \rightarrow F(\lambda)$ for any $\lambda>0$.

Obviously, all subcritical Jiřina processes satisfy assumption (A). We prove the following main results in Section 3.

Theorem 2.1. If assumption (A) holds then, for any $1>c>\gamma$, there exists a generalized random variable $Y \in\{0,+\infty\}$ such that $Y_{n} \rightarrow Y$ a.s.

Theorem 2.2. If assumption (A) holds then $Y_{n} \rightarrow+\infty$ a.s. for any $c<\gamma$.

Theorems 2.1 and 2.2 show that the speed of extinction is closely related to $\gamma$, the pseudodrift at $x=0$; for any $c \neq \gamma, c^{n}$ is not a good estimation for the speed of the continuous-state PSDBP $X$ to become extinct. A potentially good estimation is $\gamma^{n}$. However, the following two examples show that this case is complicated and subtle.

Example 2.1. Let $r \in(0,1 / \mathrm{e})$ and $\gamma(x)=\frac{1}{2}(1+1 / \ln (x \wedge r))$. Suppose that $X=\left\{X_{n}, n=\right.$ $0,1,2, \ldots\}$ is a continuous-state PSDBP with $r>X_{0}=x_{0}>0$ and RCF

$$
F(x, \lambda)=\gamma(x) \lambda
$$


In this case, $\gamma=\lim _{x \rightarrow 0} \gamma(x)=\frac{1}{2}$ and $X_{n}$ is indeed a positive sequence with

$$
X_{n+1}=X_{n} \gamma\left(X_{n}\right)=\frac{X_{n}}{2}\left(1+\frac{1}{\ln X_{n}}\right) .
$$

Let $d=\frac{1}{2}(1+1 / \ln r)$. Then $d \leq \gamma(x)<\frac{1}{2}$ for any $x>0$. Let $Y_{n}=X_{n} / \gamma^{n}=2^{n} X_{n}$. Then, for any $n \geq 0, d^{n} X_{0} \leq Y_{n} \leq X_{0}$ and

$$
Y_{n+1}=Y_{n}\left(1-\frac{1}{n \ln 2-\ln Y_{n}}\right) \leq Y_{n}\left(1-\frac{1}{n(\ln 2-\ln d)-\ln x_{0}}\right) .
$$

Therefore,

$$
0 \leq Y_{n} \leq x_{0} \prod_{k=0}^{n}\left(1-\frac{1}{k(\ln 2-\ln d)-\ln x_{0}}\right)
$$

From

$$
\sum_{k=1}^{+\infty} \frac{1}{k(\ln 2-\ln d)-\ln x_{0}}=+\infty
$$

we obtain $Y_{n} \rightarrow 0$ as $n \rightarrow+\infty$.

The following example shows that the limit random variable $Y$ of $X_{n} / \gamma^{n}$ may be proper and nondegenerate.

Example 2.2. Let $X=\left\{X_{n}\right\}$ be a continuous-state PSDBP with

$$
\begin{aligned}
F(x, \lambda) & =\frac{1-(x \wedge 0.5)}{2} \lambda+\frac{1}{2} \int_{0+}^{+\infty}\left(1-\mathrm{e}^{-\lambda s}\right) \frac{1}{(x \wedge 0.5)^{3}} \exp \left\{-\frac{s}{(x \wedge 0.5)^{2}}\right\} \mathrm{d} s \\
& =\frac{1-(x \wedge 0.5)}{2} \lambda+\frac{(x \wedge 0.5) \lambda}{2\left(1+(x \wedge 0.5)^{2} \lambda\right)}
\end{aligned}
$$

and $X_{0}=1$. Then Proposition 3.1 shows that $Y_{n}:=X_{n} / \gamma^{n}=2^{n} X_{n}$ converges a.s. to a random variable $Y \in(0,+\infty)$.

The two examples show that it is difficult to obtain a satisfactory result for the case in which $c=\gamma$ if we suppose that only assumption (A) holds. In Subsection 3.3 we briefly discuss this case under some special assumptions. In Section 4, via a more general scheme, we find some suitable sufficient conditions such that $X_{n} / \gamma^{n}$ converges a.s. to a proper, nondegenerate limit.

In Section 4 we further assume that

(B1) for any $x>0, m(x)=\gamma(x)+\int_{0+}^{+\infty} u v(x, \mathrm{~d} u)<+\infty$ and $\lim _{x \rightarrow 0} m(x)=m<1$;

(B2) there exists an $x_{0}>0$ such that $-\gamma(x) / x \leq \gamma^{\prime}(x) \leq 0$ for any $x \in\left(0, x_{0}\right)$;

(B3) there exists a measure $\eta$ on $(0,+\infty)$ with $\int_{0+}^{+\infty} u \eta(\mathrm{d} u)-\int_{0}^{1} u \ln (u) \eta(\mathrm{d} u)<+\infty$ such that, for any $x \in\left(0, x_{0}\right)$ and $s>0, v(x,[s,+\infty)) \leq \eta([s,+\infty))$.

Remark 2.1. Assumption (B3) indicates that, for any $x \in\left(0, x_{0}\right)$ and $\lambda, \beta>0$,

$$
\begin{aligned}
\int_{0+}^{+\infty}\left(1-\mathrm{e}^{-\lambda u}\right) v(x, \mathrm{~d} u) & \leq \int_{0+}^{+\infty}\left(1-\mathrm{e}^{-\lambda u}\right) \eta(\mathrm{d} u), \\
\int_{0+}^{+\infty} \frac{u}{u x+\beta} v(x, \mathrm{~d} u) & \leq \int_{0+}^{+\infty} \frac{u}{u x+\beta} \eta(\mathrm{d} u) .
\end{aligned}
$$


The main result proved in Section 4 is as follows.

Theorem 2.3. Assume that (B1)-(B3) hold. Then there exists a sequence of positive constants $\left\{c_{n}, n \geq 0\right\}$ with $\lim _{n \rightarrow+\infty} c_{n+1} / c_{n}=\gamma$ such that $Y_{n}=X_{n} / c_{n}$ converges a.s. to a proper, nondegenerate random variable.

By Theorem 2.3 we can obtain sufficient conditions for $X_{n} / \gamma^{n}$ to converge a.s. to a proper, nondegenerate limit; see Corollary 4.1, below. In addition, since assumptions (B1)-(B2) always hold for Jiřina processes, by Theorem 2.3 we obtain the following result, which, in essence, is the 'if' part of Theorem 2.2 of [8]. The proof is omitted.

Corollary 2.1. Suppose that $X=\left\{X_{n}\right\}$ is a subcritical Jiřina process with parameters $\gamma$ and $\mu(\cdot)$. If $\gamma>0$ and $\int_{0}^{1} u|\ln u| \mu(\mathrm{d} u)<+\infty$, then we can take $c_{n}=c_{0} \gamma^{n}$ for some $c_{0}>0$ and all $n \geq 0$ such that $X_{n} / c_{n}$ converges a.s. to a proper, nondegenerate random variable.

In the following example we provide a continuous-state PSDBP which satisfies all the assumptions given in this section.

Example 2.3. Let $g(s)=1 / s^{3 / 2} \wedge 1 / s^{5 / 2}$ for $s>0$. Then

$$
\int_{0+}^{+\infty} s g(s) \mathrm{d} s=\int_{0+}^{1} s^{-1 / 2} \mathrm{~d} s+\int_{1}^{+\infty} s^{-3 / 2} \mathrm{~d} s=4 .
$$

For any $x \geq 0$ and $\lambda \geq 0$, define

$$
F(x, \lambda)=\frac{\lambda}{2(1+x / 100)}+\frac{1}{6} \int_{0+}^{+\infty}\left(1-\mathrm{e}^{-\lambda s}\right) \frac{g(s)}{2+\sin (x \pi)} \mathrm{d} s .
$$

Let $X=\left\{X_{n}, n \geq 0\right\}$ be a continuous-state PSDBP determined by $F(x, \lambda)$. Then by some simple calculations we know that $X$ has the following properties.

(P1) For every $x>0, \gamma(x)=50 /(100+x) \in\left(0, \frac{1}{2}\right)$ and

$$
v(x, \mathrm{~d} s)=\frac{g(s)}{6(2+\sin (x \pi))} \mathrm{d} s .
$$

(P2) As $x \rightarrow 0, F(x, \lambda)$ converges to

$$
F(\lambda)=\frac{\lambda}{2}+\frac{1}{12} \int_{0+}^{+\infty}\left(1-\mathrm{e}^{-\lambda s}\right) g(s) \mathrm{d} s
$$

and $\gamma(x) \rightarrow \frac{1}{2}$

(P3) For $x>0$,

$$
m(x)=\frac{50}{100+x}+\frac{2}{6+3 \sin (x \pi)}<+\infty
$$

and, as $x \rightarrow 0, m(x) \rightarrow \frac{5}{6}<1$. In addition, for $x=(4 k+3) / 2$, where $0 \leq k \leq 24$, $1<m(x)<\frac{7}{6}$.

(P4) For $x \in(0,1)$,

$$
\gamma^{\prime}(x)=-\frac{50}{(100+x)^{2}}<0 \quad \text { and } \quad \gamma^{\prime}(x)>-\frac{50}{(100+x) x}=-\frac{\gamma(x)}{x} \text {. }
$$


(P5) Let $\eta(\mathrm{d} s)=\frac{1}{6} g(s) \mathrm{d} s$. Then

$$
\int_{0+}^{+\infty} s \eta(\mathrm{d} s)-\int_{0+}^{1} s \ln (s) \eta(\mathrm{d} s)=\frac{2}{3}-\frac{1}{6} \int_{0+}^{1} \frac{\ln s}{s^{1 / 2}} \mathrm{~d} s=\frac{4}{3}<+\infty,
$$

and, for any $x \in(0,1)$ and $s>0, v(x,[s,+\infty)) \leq \eta([s,+\infty))$.

(P6) From the fact that, for any $x \geq 0$ and $\lambda>0$,

$$
\int_{0+}^{+\infty}\left(\frac{1}{\lambda} \wedge s\right) v(x, \mathrm{~d} s)=\frac{1}{6} \int_{0+}^{+\infty}\left(\frac{1}{\lambda} \wedge s\right) \frac{g(s)}{2+\sin (x \pi)} \mathrm{d} s \leq \frac{1}{6} \int_{0+}^{+\infty}\left(\frac{1}{\lambda} \wedge s\right) g(s) \mathrm{d} s,
$$

by Proposition 2.4 of [5], we know that the criterion function

$$
f(x)=\sup \{\lambda \geq 0: \lambda \leq F(x, \lambda)\}
$$

is bounded. Furthermore, since $m(x)$ is bounded and $\lim \sup _{x \rightarrow+\infty} m(x) \leq \frac{2}{3}<1$, by the same proof as Corollary 3.1 of [7], we find that $X_{n} \rightarrow 0$ a.s. as $n \rightarrow \infty$.

Properties (P2)-(P6) indicate that assumptions (A) and (B1)-(B3) hold for $X$, which becomes extinct. Hence, Theorem 2.3 is true for this process.

\section{Geometric speed of extinction}

In this section we discuss the geometric speed of extinction. First, recall that $X_{n} \rightarrow 0$ a.s. implies that, for any $\delta>0$,

$$
\lim _{n \rightarrow+\infty} \mathrm{P}\left(\bigcap_{m=n}^{+\infty}\left\{X_{m} \leq \delta\right\}\right)=1 .
$$

The main tool used in this section is exponent martingales.

\subsection{The case in which $c>\gamma$}

In this subsection we will prove Theorem 2.1 and obtain some interesting corollaries.

Proof of Theorem 2.1. It is sufficient to prove that, for a given $\lambda>0, \exp \left\{-\lambda Y_{n}\right\} \rightarrow \tilde{Y}:=$ $\mathrm{e}^{-\lambda Y}$ a.s., and that $\mathrm{P}(\tilde{Y}=0)+\mathrm{P}(\tilde{Y}=1)=1$. We divide the proof into four steps.

Step 1. There exists a $\lambda_{0}>0$ such that, for any given $\lambda>\lambda_{0}$, we have $\delta(\lambda)>0$ satisfying

$$
\frac{\gamma(x)}{c} \lambda+c^{n} f\left(x, \frac{\lambda}{c^{n+1}}\right) \leq \lambda
$$

for all $0 \leq x<\delta(\lambda)$ and $n \geq 0$. Here, $f(x, \lambda):=\int_{0+}^{+\infty}\left(1-\mathrm{e}^{-\lambda s}\right) v(x, \mathrm{~d} s)$.

Let

$$
\beta=\frac{c+\gamma}{2 c} \text { and } \phi(\lambda)=\beta \lambda+\int_{0+}^{+\infty}\left(1-\mathrm{e}^{-\lambda s / c}\right) \mu(\mathrm{d} s) .
$$

Since $\lim _{\lambda \rightarrow+\infty} \phi(\lambda) / \lambda=\beta<1$, there exists a $\lambda_{0}>0$ such that, for any $\lambda>\lambda_{0}, \phi(\lambda) \leq \lambda$. For a given $\lambda>\lambda_{0}$, since $\lim _{x \rightarrow 0} F(x, \lambda / c)=F(\lambda / c)$, there exists a constant $\delta(\lambda)>0$ such that, for any $x \leq \delta(\lambda)$,

$$
F\left(x, \frac{\lambda}{c}\right) \leq F\left(\frac{\lambda}{c}\right)+\frac{c-\gamma}{2 c}=\phi(\lambda) \leq \lambda .
$$


Note that, for any $n \geq 0, c^{n} f\left(x, \lambda / c^{n+1}\right) \leq f(x, \lambda / c)$ for $c<1$. Hence, from (3.3) we obtain

$$
\frac{\gamma(x)}{c} \lambda+c^{n} f\left(x, \frac{\lambda}{c^{n+1}}\right) \leq \frac{\gamma(x)}{c} \lambda+f\left(x, \frac{\lambda}{c}\right)=F\left(x, \frac{\lambda}{c}\right) \leq \lambda .
$$

In the remainder of the proof we let $\lambda>\lambda_{0}$ be a fixed constant and we suppress the notation $\lambda$ in $\delta(\lambda)$.

Step 2. For any $k, n \geq 0$, let $\mathcal{F}_{k}^{(n)}=\mathcal{F}\left\{X_{n}, X_{n+1}, \ldots, X_{n+k}\right\}$ be the $\sigma$-algebra generated by $\left\{X_{n}, X_{n+1}, \ldots, X_{n+k}\right\}$, and let

$$
A_{k}^{(n)}=\bigcap_{m=n}^{n+k}\left\{X_{m} \leq \delta\right\} \quad \text { and } \quad \tilde{Y}_{k+1}^{(n)}=\mathbf{1}_{A_{k}^{(n)}} Y_{n+k+1},
$$

where $\mathbf{1}_{A_{k}^{(n)}}$ is the indicator function of the set $A_{k}^{(n)}$. Then $\left\{\exp \left\{-\lambda \tilde{Y}_{k}^{(n)}\right\}, k=1,2, \ldots\right\}$ is the bounded, nonnegative submartingale with respect to $\left\{\mathcal{F}_{k}^{(n)}, k=1,2, \ldots\right\}$ for any $n \geq 0$.

By the definition of $\tilde{Y}_{k+1}^{(n)}$, we have

$$
\begin{aligned}
\mathrm{E}\left[\exp \left\{-\lambda \tilde{Y}_{k+1}^{(n)}\right\} \mid \mathcal{F}_{k}^{(n)}\right] & =\mathrm{E}\left[\mathbf{1}_{A_{k}^{(n)}} \exp \left\{-\lambda \tilde{Y}_{k+1}^{(n)}\right\} \mid \mathcal{F}_{k}^{(n)}\right]+\mathbf{1}_{\bar{A}_{k}^{(n)}} \\
& =\mathbf{1}_{A_{k}^{(n)}} \mathrm{E}\left[\exp \left\{-\lambda Y_{n+k+1}\right\} \mid \mathcal{F}_{k}^{(n)}\right]+\mathbf{1}_{\bar{A}_{k}^{(n)}},
\end{aligned}
$$

where $\bar{A}_{k}^{(n)}$ is the complementary set of $A_{k}^{(n)}$. Note that, for any $n>0$,

$$
\begin{aligned}
\mathrm{E}\left[\exp \left\{-\lambda Y_{n+k+1}\right\} \mid \mathcal{F}_{k}^{(n)}\right] & =\mathrm{E}\left[\exp \left\{-\frac{\lambda X_{n+k+1}}{c^{n+k+1}}\right\} \mid X_{n+k}\right] \\
& =\exp \left\{-Y_{n+k} G_{n+k}\left(X_{n+k}, \lambda\right)\right\},
\end{aligned}
$$

where

$$
G_{n+k}\left(X_{n+k}, \lambda\right)=\gamma\left(X_{n+k}\right) \frac{\lambda}{c}+\int_{0+}^{+\infty} c^{n+k}\left(1-\exp \left\{-\frac{\lambda s}{c^{n+k+1}}\right\}\right) v\left(X_{n+k}, \mathrm{~d} s\right)
$$

Obviously, (3.2) shows that

$$
\mathbf{1}_{A_{k}^{(n)}} G_{n+k}\left(X_{n+k}, \lambda\right) \leq \lambda .
$$

From the fact that $A_{k}^{(n)} \subset A_{k-1}^{(n)}$ for any $k>1$, we have

$$
\mathbf{1}_{A_{k}^{(n)}} Y_{n+k}=\mathbf{1}_{A_{k}^{(n)}} \tilde{Y}_{k}^{(n)}
$$

Then (3.4)-(3.7) imply that

$$
\mathrm{E}\left[\exp \left\{-\lambda \tilde{Y}_{k+1}^{(n)}\right\} \mid \mathcal{F}_{k}^{(n)}\right] \geq \exp \left\{-\lambda \tilde{Y}_{k}^{(n)}\right\},
$$

which is the desired conclusion.

Step 3. We prove that $\exp \left\{-\lambda Y_{n}\right\}$ converges a.s. to a random variable $\tilde{Y} \in[0,1]$. 
By the submartingale convergence theorem, there exists a random variable $\tilde{W}_{n} \in[0,1]$ such that, for any $n, \exp \left\{-\lambda \tilde{Y}_{k+1}^{(n)}\right\} \rightarrow \tilde{W}_{n}$ a.s. as $k \rightarrow+\infty$. By the definition of $\tilde{Y}_{k}^{(n)}, \tilde{Y}_{k}^{(n+1)} \geq \tilde{Y}_{k+1}^{(n)}$ for any $k>0$ and $n>0$, which implies that $\tilde{W}_{n} \geq \tilde{W}_{n+1}$ a.s. for any $n>0$. Consequently, there exists a random variable $\tilde{Y} \in[0,1]$ such that $\tilde{W}_{n} \rightarrow \tilde{Y}$ a.s. as $n \rightarrow+\infty$, which means that, for any $\delta>0$ and any $\varepsilon>0$, there exists an $n_{1}>0$ such that, for any $n \geq n_{1}$,

$$
\mathrm{P}\left(\left|\tilde{W}_{n}-\tilde{Y}\right|>\frac{\delta}{3}\right)<\frac{\varepsilon}{3} \text {. }
$$

Taking $n>n_{1}$, by the fact that

$$
\left\{\left|\exp \left\{-\lambda Y_{n+k}\right\}-\exp \left\{-\lambda \tilde{Y}_{k}^{(n)}\right\}\right|>0\right\} \subset \bigcup_{m=n}^{n+k-1}\left\{X_{m}>\delta\right\},
$$

we know that

$$
\mathrm{P}\left(\bigcup_{k=1}^{+\infty}\left\{\left|\exp \left\{-\lambda Y_{n+k}\right\}-\exp \left\{-\lambda \tilde{Y}_{k}^{(n)}\right\}\right|>0\right\}\right) \leq \mathrm{P}\left(\bigcup_{m=n}^{+\infty}\left\{X_{m}>\delta\right\}\right) .
$$

Combining (3.1) and (3.9), there exists an $n_{2}>n_{1}$ such that

$$
\mathrm{P}\left(\bigcup_{k=1}^{+\infty}\left\{\left|\exp \left\{-\lambda Y_{k+n_{2}}\right\}-\exp \left\{-\lambda \tilde{Y}_{k}^{\left(n_{2}\right)}\right\}\right|>0\right\}\right) \leq \frac{\varepsilon}{3} .
$$

Furthermore, $\exp \left\{-\lambda \tilde{Y}_{k}^{\left(n_{2}\right)}\right\} \rightarrow \tilde{W}_{n_{2}}$ a.s. implies that

$$
\mathrm{P}\left(\bigcup_{k=n_{3}}^{+\infty}\left\{\left|\exp \left\{-\lambda \tilde{Y}_{k}^{\left(n_{2}\right)}\right\}-\tilde{W}_{n_{2}}\right|>\frac{\delta}{3}\right\}\right)<\frac{\varepsilon}{3} \quad \text { for some } n_{3}>0 .
$$

Let $K=n_{2}+n_{3}$. Define

$$
\begin{gathered}
A:=\bigcup_{m=n_{3}}^{+\infty}\left\{\left|\exp \left\{-\lambda Y_{n_{2}+m}\right\}-\exp \left\{-\lambda \tilde{Y}_{m}^{\left(n_{2}\right)}\right\}\right|>\frac{\delta}{3}\right\}, \\
B:=\bigcup_{m=n_{3}}^{+\infty}\left\{\left|\exp \left\{-\lambda \tilde{Y}_{m}^{\left(n_{2}\right)}\right\}-\tilde{W}_{n_{2}}\right|>\frac{\delta}{3}\right\},
\end{gathered}
$$

and

$$
C:=\left\{\left|\tilde{W}_{n_{2}}-\tilde{Y}\right|>\frac{\delta}{3}\right\}
$$

Then

$$
\mathrm{P}\left(\bigcup_{m=K}^{+\infty}\left\{\left|\exp \left\{-\lambda Y_{m}\right\}-\tilde{Y}\right|>\delta\right\}\right) \leq \mathrm{P}(A \cup B \cup C) \leq \mathrm{P}(A)+\mathrm{P}(B)+\mathrm{P}(C) .
$$

From (3.8), (3.10), and (3.11), we obtain

$$
\mathrm{P}\left(\bigcup_{m=K}^{+\infty}\left\{\left|\exp \left\{-\lambda Y_{m}\right\}-\tilde{Y}\right|>\delta\right\}\right)<\varepsilon
$$

which implies that $\exp \left\{-\lambda Y_{n}\right\}$ converges a.s. to $\tilde{Y}$ as $n \rightarrow+\infty$. 
Step 4. We prove that $\tilde{Y} \in\{0,1\}$ a.s., i.e. $\mathrm{P}(\tilde{Y}=0)+\mathrm{P}(\tilde{Y}=1)=1$.

By an argument similar to that used to prove (3.5), we have

$$
\mathrm{E}\left[\exp \left\{-\lambda Y_{n+1}\right\}\right]=\mathrm{E}\left[\exp \left\{-Y_{n} G_{n}\left(X_{n}, \lambda\right)\right\}\right]=\mathrm{E}\left[\left(\exp \left\{-\lambda Y_{n}\right\}\right)^{G_{n}\left(X_{n}, \lambda\right) / \lambda}\right],
$$

where

$$
\frac{G_{n}\left(X_{n}, \lambda\right)}{\lambda}=\frac{\gamma\left(X_{n}\right)}{c}+\lambda^{-1} c^{n} f\left(X_{n}, \frac{\lambda}{c^{n+1}}\right) .
$$

Note that, for any $\varepsilon>0$,

$$
\begin{aligned}
c^{n} f\left(x, \frac{\lambda}{c^{n+1}}\right) & =\int_{0+}^{+\infty} \frac{c^{n}\left(1-\exp \left\{-\lambda s / c^{n+1}\right\}\right)}{1-\mathrm{e}^{-\lambda s / c}}\left(1-\mathrm{e}^{-\lambda s / c}\right) v(x, \mathrm{~d} s) \\
& \leq \int_{(\varepsilon,+\infty)} \frac{c^{n}}{1-\mathrm{e}^{-\lambda \varepsilon / c}}\left(1-\mathrm{e}^{-\lambda s / c}\right) v(x, \mathrm{~d} s)+\int_{(0, \varepsilon]}\left(1-\mathrm{e}^{-\lambda s / c}\right) v(x, \mathrm{~d} s) \\
& \leq \frac{c^{n}}{1-\mathrm{e}^{-\lambda \varepsilon / c}} f\left(x, \frac{\lambda}{c}\right)+\int_{(0, \varepsilon]}\left(1-\mathrm{e}^{-\lambda s / c}\right) v(x, \mathrm{~d} s) .
\end{aligned}
$$

By assumption (A), we easily find that $\left(1-\mathrm{e}^{-\lambda s}\right) \nu(x, \mathrm{~d} s)$ converges weakly to $\left(1-\mathrm{e}^{-\lambda s}\right) \mu(\mathrm{d} s)$ as $x \rightarrow 0$. Hence,

$$
\limsup _{x \rightarrow 0} \int_{(0, \varepsilon]}\left(1-\mathrm{e}^{-\lambda s}\right) v(x, \mathrm{~d} s) \leq \int_{(0, \varepsilon]}\left(1-\mathrm{e}^{-\lambda s}\right) \mu(\mathrm{d} s) .
$$

For any $x_{n} \rightarrow 0$, from (3.13), it follows that

$$
\begin{aligned}
\limsup _{n \rightarrow+\infty} c^{n} f\left(x_{n}, \frac{\lambda}{c^{n+1}}\right) & \leq \limsup _{n \rightarrow+\infty} \int_{(0, \varepsilon]}\left(1-\mathrm{e}^{-\lambda s / c}\right) \nu\left(x_{n}, \mathrm{~d} s\right) \\
& \leq \int_{(0, \varepsilon]}\left(1-\mathrm{e}^{-\lambda s / c}\right) \mu(\mathrm{d} s) \\
& \rightarrow 0 \quad \text { as } \varepsilon \rightarrow 0 .
\end{aligned}
$$

Since $X_{n} \rightarrow 0$ a.s., (3.14) implies that

$$
c^{n} f\left(X_{n}, \frac{\lambda}{c^{n+1}}\right) \rightarrow 0 \text { a.s. }
$$

Consequently, $G_{n}\left(X_{n}, \lambda\right) / \lambda \rightarrow \gamma / c$ a.s. as $n \rightarrow+\infty$, and

$$
\left(\exp \left\{-\lambda Y_{n}\right\}\right)^{G_{n}\left(X_{n}, \lambda\right) / \lambda} \rightarrow \tilde{Y}(\lambda)^{\gamma / c} \text { a.s. }
$$

Combining (3.12) and (3.15), we obtain

$$
\mathrm{E}[\tilde{Y}]=\mathrm{E}\left[\tilde{Y}^{\gamma / c}\right]
$$

Since $\gamma / c<1,(3.16)$ implies that $\mathrm{P}(0<\tilde{Y}<1)=0$. The proof of step 4 and, hence, of the theorem is now complete.

Corollary 3.1. Under assumption (A), if in a neighborhood of 0 the offspring mean function $m(x)$ exists and $m(x) \rightarrow m<1$ as $x \rightarrow 0$, then, for any $c>m, X_{n} / c^{n} \rightarrow 0$ a.s. as $n \rightarrow+\infty$. 
Proof. From Theorem 2.1 we know that $X_{n} / c^{n} \rightarrow Y \in\{0,+\infty\}$ a.s. Suppose that $p=$ $\mathrm{P}\{Y=+\infty\}>0$. Since $m(x) \rightarrow m<1$ and $c>m$, there exists a constant $\delta>0$ such that, for any $x<\delta, m(x)<c$. When $p>0$, by (3.1), there exists a positive integer $N$ which may depend on $\delta$ and $p / 2$ such that

$$
\mathrm{P}\left(\bigcap_{m=N}^{+\infty}\left\{X_{m} \leq \delta\right\}\right)>1-\frac{p}{2} .
$$

For any $n \geq 0$, let $A_{N}^{(n)}=\bigcap_{m=N}^{N+n}\left\{X_{m} \leq \delta\right\}, A_{N}=\bigcap_{m=N}^{+\infty}\left\{X_{m} \leq \delta\right\}$, and

$$
\mathcal{F}_{N}^{(n)}=\mathscr{F}\left\{X_{N}, X_{N+1}, \ldots, X_{N+n}\right\} .
$$

Then

$$
W_{n}:=\mathbf{1}_{A_{N}^{(n)}} \frac{X_{n}}{c^{n}} \rightarrow W:=\mathbf{1}_{A_{N}} Y .
$$

Hence, $\mathrm{P}(W=+\infty)>p / 2>0$. On the other hand, by (1.3) we have

$$
\begin{aligned}
\mathrm{E}\left[W_{n+1}\right] & =\mathrm{E}\left[\mathrm{E}\left[\mathbf{1}_{A_{N}^{(n+1)}} \frac{X_{N+n+1}}{c^{N+n+1}} \mid \mathcal{F}_{N}^{(n)}\right]\right] \\
& \leq \mathrm{E}\left[\mathrm{E}\left[\mathbf{1}_{A_{N}^{(n)}} \frac{X_{N+n+1}}{c^{N+n+1}} \mid \mathcal{F}_{N}^{(n)}\right]\right] \\
& =\mathrm{E}\left[\mathbf{1}_{A_{N}^{(n)}} \frac{m\left(X_{N+n}\right) X_{N+n}}{c^{N+n+1}}\right] \\
& \leq \mathrm{E}\left[\mathbf{1}_{A_{N}^{(n)}} \frac{X_{N+n}}{c^{N+n}}\right] \\
& \leq \cdots \\
& \leq \mathrm{E}\left[\mathbf{1}_{A_{N}^{(0)}} \frac{X_{N}}{c^{N}}\right] \\
& \leq \frac{\delta}{c^{N}} \quad \text { for any } n \geq 0 . \\
& <+\infty \quad
\end{aligned}
$$

By Fatou's lemma, we find that $\mathrm{E}[W]<+\infty$, which contradicts the fact that $\mathrm{P}(W=+\infty)>0$. Consequently, $p=0$. That is, $X_{n} / c^{n} \rightarrow 0$ a.s.

Corollary 3.2. Suppose that assumption (A) holds. If in a neighborhood of 0 the offspring mean function $m(x)$ exists and, as $x \rightarrow 0, m(x)$ tends in a monotone fashion from below to a constant $m$ with $\gamma<m<1$, then $X_{n} / m^{n} \rightarrow 0$ a.s. as $n \rightarrow+\infty$.

Proof. The proof is similar to that of Corollary 3.1. We omit it here.

Remark 3.1. Theorem 2.1 indicates that, for any $c$ larger than the pseudo-drift at $x=0$, $\left\{c^{n}, n \geq 0\right\}$ is not a sequence of suitable parameters to estimate the speed of extinction. Corollary 3.1 and Corollary 3.2 show that the pseudo-mean at $x=0$ is an upper bound for the geometric speed of extinction. 


\subsection{The case in which $c<\gamma$}

Now we prove Theorem 2.2, which in essence discusses the lower bound for the geometric speed of extinction.

Proof of Theorem 2.2. The proof is very similar to that of Theorem 2.1. For any $\lambda>0$, let $\beta=(\gamma+c) / 2 c$. From assumption (A), it follows that there exists a $\delta>0$ such that

$$
\frac{\gamma(x)}{c} \lambda \geq \beta \lambda>\lambda \quad \text { for any } x \leq \delta .
$$

Let $\tilde{Y}_{0}^{(n)}=Y_{n}$,

$$
\tilde{Y}_{k+1}^{(n)}(\omega)= \begin{cases}Y_{n+k+1}(\omega), & \omega \in A_{k}^{(n)}, \\ \tilde{Y}_{k}^{(n)}(\omega), & \omega \in \bar{A}_{k}^{(n)},\end{cases}
$$

where $A_{k}^{(n)}=\bigcap_{m=n}^{n+k}\left\{X_{m} \leq \delta\right\}$, and let $\mathcal{F}_{k}^{(n)}=\mathcal{F}\left\{X_{n}, X_{n+1}, \ldots, X_{n+k}\right\}$. Then

$$
\mathrm{E}\left[\exp \left\{-\lambda \tilde{Y}_{k+1}^{(n)}\right\} \mid \mathcal{F}_{k}^{(n)}\right]=\mathrm{E}\left[\mathbf{1}_{A_{k}^{(n)}} \exp \left\{-\lambda Y_{n+k+1}\right\} \mid \mathcal{F}_{k}^{(n)}\right]+\mathbf{1}_{\bar{A}_{k}^{(n)}} \exp \left\{-\lambda \tilde{Y}_{k}^{(n)}\right\} .
$$

Note that $Y_{n}=X_{n} / c^{n}$ for any $n>0$. Hence,

$$
\begin{aligned}
& \mathrm{E}\left[\exp \left\{-\lambda Y_{n+k+1}\right\} \mid \mathcal{F}_{k}^{(n)}\right] \\
& \quad=\exp \left\{-Y_{n+k}\left(\gamma\left(X_{n+k}\right) \frac{\lambda}{c}+\int_{0+}^{+\infty} c^{k+n}\left(1-\exp \left\{-\frac{\lambda s}{c^{k+n+1}}\right\} v\left(X_{n+k}, \mathrm{~d} s\right)\right)\right\}\right. \\
& \quad \leq \exp \left\{-Y_{n+k} \gamma\left(X_{n+k}\right) \frac{\lambda}{c}\right\} .
\end{aligned}
$$

Combining (3.18) and (3.19), we obtain

$$
\mathrm{E}\left[\exp \left\{-\lambda \tilde{Y}_{k+1}^{(n)}\right\} \mid \mathcal{F}_{k}^{(n)}\right] \leq \mathbf{1}_{A_{k}^{(n)}} \exp \left\{-\tilde{Y}_{k}^{(n)} \gamma\left(X_{n+k}\right) \frac{\lambda}{c}\right\}+\mathbf{1}_{\bar{A}_{k}^{(n)}} \exp \left\{-\lambda \tilde{Y}_{k}^{(n)}\right\} .
$$

Then (3.17) and (3.20) imply that

$$
\mathrm{E}\left[\exp \left\{-\lambda \tilde{Y}_{k+1}^{(n)}\right\} \mid \mathcal{F}_{k}^{(n)}\right] \leq \exp \left\{-\lambda \tilde{Y}_{k}^{(n)}\right\},
$$

which shows that $\left\{\exp \left\{-\lambda \tilde{Y}_{k+1}^{(n)}\right\}, k=1,2, \ldots\right\}$ is the bounded, nonnegative supermartingale with respect to $\left\{\mathcal{F}_{k}^{(n)}, k=1,2, \ldots\right\}$ for any $n>0$. By the supermartingale convergence theorem, there exists a random variable $\tilde{W}_{n} \in[0,1]$ such that

$$
\exp \left\{-\lambda \tilde{Y}_{k+1}^{(n)}\right\} \rightarrow \tilde{W}_{n} \quad \text { a.s. }
$$

as $k \rightarrow+\infty$ for any $n$. According to the definition of $\tilde{Y}_{k}^{(n)}$,

$$
\tilde{Y}_{k}^{(n+1)}=\tilde{Y}_{k+1}^{(n)}
$$

on $A_{k}^{(n)}$ for any $k>0$ and $n>0$. Therefore,

$$
\tilde{W}_{n}=\tilde{W}_{n+1} \quad \text { a.s. on } \quad A^{(n)}:=\bigcap_{m=n}^{+\infty}\left\{X_{m} \leq \delta\right\} \quad \text { for any } n>0 .
$$


Let $B_{1}=A^{(1)}, B_{n}=A^{(n)} \backslash A^{(n-1)}, n \geq 2$. Then

$$
\bigcup_{i=1}^{+\infty} B_{i}=\lim _{n \rightarrow+\infty} \bigcup_{i=1}^{n} B_{i}=\lim _{n \rightarrow+\infty} A^{(n)}=\lim _{n \rightarrow+\infty} \bigcap_{m=n}^{+\infty}\left\{X_{m} \leq \delta\right\} .
$$

Define

$$
\tilde{W}=\sum_{k=1}^{+\infty} \mathbf{1}_{B_{k}} \tilde{W}_{k}=\sum_{k=n+1}^{+\infty} \mathbf{1}_{B_{k}} \tilde{W}_{k}+\mathbf{1}_{A^{(n)}} \tilde{W}_{n}
$$

Obviously, for any $\varepsilon>0$,

$$
\mathrm{P}\left(\bigcap_{m=n}^{+\infty}\left\{\left|\tilde{W}_{m}-\tilde{W}\right| \leq \varepsilon\right\}\right) \geq \mathrm{P}\left(\bigcap_{m=n}^{+\infty} A^{(m)}\right)=\mathrm{P}\left(A^{(n)}\right) \rightarrow 1 \quad \text { as } n \rightarrow+\infty .
$$

This means that $\tilde{W}_{k} \rightarrow \tilde{W}$ a.s. Then by similar arguments to those used in step 3 of the proof of Theorem 2.1 we obtain

$$
\exp \left\{-\lambda Y_{n}\right\} \rightarrow \tilde{W} \geq 0 \quad \text { a.s. }
$$

Consequently, for any $n \geq 0$, from (3.17), it follows that

$$
\begin{aligned}
\mathrm{E}[\tilde{W}]= & \lim _{k \rightarrow+\infty} \mathrm{E}\left[\exp \left\{-\lambda Y_{n+1+k}\right\}\right] \\
= & \lim _{k \rightarrow+\infty} \mathrm{E}\left[\operatorname { e x p } \left\{-Y_{n+k}\left(\frac{\lambda \gamma\left(x_{n+1}\right)}{c}\right.\right.\right. \\
& \left.\left.\left.\quad+\int_{0+}^{+\infty} c^{n}\left(1-\exp \left\{\frac{\lambda s}{c^{n+1}}\right\}\right) v\left(x_{n+k}, \mathrm{~d} s\right)\right)\right\}\right] \\
\leq & \lim _{k \rightarrow+\infty} \mathrm{E}\left[\mathbf{1}_{A_{k}^{(n)}} \exp \left\{-\beta \lambda Y_{n+k}\right\}\right]+\mathrm{P}\left(\bar{A}_{k}^{(n)}\right) .
\end{aligned}
$$

Since $A_{k}^{(n)} \subset A_{k-1}^{(n)}$ for any $n \geq 1$ and $k \geq 1$, we have, similarly,

$$
\mathrm{E}\left[\mathbf{1}_{A_{k}^{(n)}} \exp \left\{-\beta \lambda Y_{n+k}\right\}\right] \leq \mathrm{E}\left[\mathbf{1}_{A_{k-1}^{(n)}} \exp \left\{-\beta \lambda Y_{n+k}\right\}\right] \leq \mathrm{E}\left[\mathbf{1}_{A_{k-1}^{(n)}} \exp \left\{-\beta^{2} \lambda Y_{n+k-1}\right\}\right]
$$

Therefore, from (3.21) and (3.22), by induction we obtain, as $n \rightarrow+\infty$,

$$
\mathrm{E}[\tilde{W}] \leq \lim _{k \rightarrow+\infty}\left(\mathrm{E}\left[\mathbf{1}_{A_{0}^{(n)}} \exp \left\{-\beta^{k} \lambda Y_{n}\right\}\right]+\mathrm{P}\left(\bar{A}_{k}^{(n)}\right)\right)=\mathrm{P}\left(\bigcup_{m=n}^{+\infty}\left\{X_{m} \geq \delta\right\}\right),
$$

where the right-hand side goes to 0 because $X_{n}>0$ a.s. and $\beta>1$. Hence, $\mathrm{E}[\tilde{W}]=0$. This implies that $Y_{n} \rightarrow+\infty$ a.s.

\subsection{The case in which $c=\gamma$}

In this subsection we study the case in which $c=\gamma$. This case is more complicated than those discussed above. Here, we only deal with some simple subcases.

Theorem 3.1. Suppose that $F(x, \lambda) \geq F(\lambda)$ for any $x>0$ and $\lambda>0$. If $F(x, \lambda) \rightarrow F(\lambda)$ as $x \rightarrow 0$ for any $\lambda>0$ then $X_{n} / \gamma^{n} \rightarrow Y \in(0,+\infty]$ a.s. 
Proof. From the fact that

$$
\begin{aligned}
& \mathrm{E}\left[\exp \left\{-\lambda \frac{X_{n+1}}{\gamma^{n+1}}\right\} \mid X_{n}\right] \\
& \quad=\exp \left\{-X_{n}\left(\gamma\left(X_{n}\right) \frac{\lambda}{\gamma^{n+1}}+\int_{0+}^{+\infty}\left(1-\exp \left\{-\frac{\lambda}{\gamma^{n+1}} s\right\}\right) \nu\left(X_{n} \mathrm{~d} s\right)\right)\right\} \\
& \quad=\exp \left\{-X_{n} F\left(X_{n}, \frac{\lambda}{\gamma^{n+1}}\right)\right\} \\
& \quad \leq \exp \left\{-X_{n} F\left(\frac{\lambda}{\gamma^{n+1}}\right)\right\} \\
& \quad \leq \exp \left\{-\lambda \frac{X_{n}}{\gamma^{n}}\right\}
\end{aligned}
$$

we easily find from the supermartingale convergence theorem that there exists a random variable $Y \in[0,+\infty]$ such that $X_{n} / \gamma^{n} \rightarrow Y$ a.s. as $n \rightarrow+\infty$. Then, for any $\lambda \geq 0$,

$$
\mathrm{E}\left[\mathrm{e}^{-\lambda Y}\right]=\lim _{n \rightarrow+\infty} \mathrm{E}\left[\exp \left\{-\lambda \frac{X_{n}}{\gamma^{n}}\right\}\right] \leq \mathrm{E}\left[\exp \left\{-\lambda \frac{X_{1}}{\gamma}\right\}\right] .
$$

Let $\lambda \rightarrow+\infty$. We have

$$
\mathrm{P}(Y=0) \leq \lim _{\lambda \rightarrow+\infty} \mathrm{E}\left[\mathrm{e}^{-\lambda Y}\right] \leq \lim _{\lambda \rightarrow+\infty} \mathrm{E}\left[\exp \left\{-\lambda \frac{X_{1}}{\gamma}\right\}\right]=0 .
$$

This implies that we can take $Y \in(0,+\infty]$ such that $X_{n} / \gamma^{n} \rightarrow Y$ a.s.

Similarly, we have the following result.

Theorem 3.2. (i) If $\gamma(x) \geq \gamma$ for any $x>0$ then $X_{n} / \gamma^{n} \rightarrow Y \in(0,+\infty]$ a.s.

(ii) If $F(x, \lambda) \leq \gamma \lambda$ for all $x>0$ and $\lambda>0$, then $X_{n} / \gamma^{n} \rightarrow Y \in[0,+\infty)$ a.s.

Proof. (i) The proof is the same as that of Theorem 3.1, except that (3.23) is replaced by

$$
\begin{aligned}
& \mathrm{E}\left[\exp \left\{-\lambda \frac{X_{n+1}}{\gamma^{n+1}}\right\} \mid X_{n}\right] \\
& \quad=\exp \left\{-X_{n}\left(\gamma\left(X_{n}\right) \frac{\lambda}{\gamma^{n+1}}+\int_{0+}^{+\infty}\left(1-\exp \left\{-\frac{\lambda}{\gamma^{n+1}} s\right\}\right) \nu\left(X_{n} \mathrm{~d} s\right)\right)\right\} \\
& \quad \leq \exp \left\{-X_{n} \gamma\left(X_{n}\right) \frac{\lambda}{\gamma^{n+1}}\right\} \\
& \quad \leq \exp \left\{-\lambda \frac{X_{n}}{\gamma^{n}}\right\} .
\end{aligned}
$$


(ii) Note that

$$
\begin{aligned}
& \mathrm{E}\left[\exp \left\{-\lambda \frac{X_{n+1}}{\gamma^{n+1}}\right\} \mid X_{n}\right] \\
& \quad=\exp \left\{-X_{n}\left(\gamma\left(X_{n}\right) \frac{\lambda}{\gamma^{n+1}}+\int_{0+}^{+\infty}\left(1-\exp \left\{-\frac{\lambda}{\gamma^{n+1}} s\right\}\right) \nu\left(X_{n} \mathrm{~d} s\right)\right)\right\} \\
& \quad=\exp \left\{-X_{n} F\left(X_{n}, \frac{\lambda}{\gamma^{n+1}}\right)\right\} \\
& \quad \geq \exp \left\{-\lambda \frac{X_{n}}{\gamma^{n}}\right\} .
\end{aligned}
$$

By the submartingale convergence theorem, there exists a random variable $Y \in[0,+\infty]$ such that $X_{n} / \gamma^{n} \rightarrow Y$ a.s. as $n \rightarrow+\infty$. Then, for any $\lambda>0$,

$$
\mathrm{E}\left[\mathrm{e}^{-\lambda Y}\right]=\lim _{n \rightarrow+\infty} \mathrm{E}\left[\exp \left\{-\lambda \frac{X_{n}}{\gamma^{n}}\right\}\right] \geq \mathrm{E}\left[\exp \left\{-\lambda \frac{X_{1}}{\gamma}\right\}\right] .
$$

Let $\lambda \rightarrow 0$. We have

$$
\mathrm{P}(Y=+\infty)=\left(1-\lim _{\lambda \rightarrow 0} \mathrm{E}\left[\mathrm{e}^{-\lambda Y}\right]\right) \leq\left(1-\lim _{\lambda \rightarrow 0} \mathrm{E}\left[\exp \left\{-\lambda \frac{X_{1}}{\gamma}\right\}\right]\right)=0
$$

This implies that we can take $Y \in[0,+\infty)$ such that $X_{n} / \gamma^{n} \rightarrow Y$ a.s.

To end this section, we explain why $Y_{n}:=X_{n} / \gamma^{n}=2^{n} X_{n}$ in Example 2.2 converges a.s. to $Y \in(0,+\infty)$. We state it as a proposition.

Proposition 3.1. The limit random variable $Y$ in Example 2.2 is proper and nondegenerate, i.e. $\mathrm{P}(0<Y<+\infty)=1$.

Proof. From $\gamma(x):=(1-(x \wedge 0.5)) / 2>0$, it follows that $X_{n}>0$ a.s. for any $n \geq 0$. In addition, $F(x, \lambda) \leq \lambda / 2$ for all $x>0, \lambda \geq 0$, and $\gamma(x) \rightarrow \frac{1}{2}$ as $x \rightarrow 0$. Therefore, from Theorem 3.2, $Y_{n}$ converges a.s. to some random variable $Y \in[0,+\infty)$. Moreover, from (2.1) we obtain the offspring mean function of $X$ as follows:

$$
m(x)=\left.F_{\lambda}^{\prime}(x, \lambda)\right|_{\lambda=0}=\frac{1-(x \wedge 0.5)}{2}+\left.\frac{(x \wedge 0.5)}{2\left(1+(x \wedge 0.5)^{2} \lambda\right)^{2}}\right|_{\lambda=0}=\frac{1}{2}
$$

for all $x>0$. Hence, from (1.3), it follows that, for any $n \geq 0$,

$$
\mathrm{E}\left[X_{n}\right]=\frac{1}{2^{n}} \mathrm{E}\left[X_{0}\right]=\frac{1}{2^{n}} .
$$

Let $W_{n}=2^{n / 2} X_{n}$. Corollary 3.1 indicates that

$$
W_{n} \rightarrow 0 \quad \text { a.s. as } n \rightarrow+\infty \text {. }
$$

For any $n, m \geq 0$, define

$$
A_{n}^{m}=\bigcap_{k=n}^{n+m}\left\{W_{n} \leq 1\right\}=\bigcap_{k=n}^{n+m}\left\{X_{k} \leq \frac{1}{2^{k / 2}}\right\} .
$$


Let $A_{n}^{\infty}=\lim _{m \rightarrow+\infty} A_{n}^{m}$. Equation (3.24) implies that

$$
\lim _{n \rightarrow+\infty} \mathrm{P}\left(A_{n}^{\infty}\right)=\lim _{n \rightarrow+\infty} \mathrm{P}\left(\bigcap_{k=n}^{+\infty}\left\{W_{k} \leq 1\right\}\right)=\lim _{n \rightarrow+\infty} \mathrm{P}\left(\bigcap_{k=n}^{+\infty}\left\{X_{k} \leq \frac{1}{2^{k / 2}}\right\}\right)=1 .
$$

If $\mathrm{P}(Y=0)=p>0$ then, from (3.25), for any $\lambda>0$ and sufficiently large $n$,

$$
\begin{aligned}
\frac{p}{2} & \leq \mathrm{E}\left[\mathrm{e}^{-\lambda Y} \mathbf{1}_{A_{n}^{\infty}}\right] \\
& =\lim _{m \rightarrow+\infty} \mathrm{E}\left[\exp \left\{-\lambda Y_{n+m+1}\right\} \mathbf{1}_{A_{n}^{m+1}}\right] \\
& \leq \lim _{m \rightarrow+\infty} \mathrm{E}\left[\exp \left\{-\lambda Y_{n+m+1}\right\} \mathbf{1}_{A_{n}^{m}}\right] \\
& =\lim _{m \rightarrow+\infty} \mathrm{E}\left[\mathrm{E}\left[\exp \left\{-\lambda Y_{n+m+1}\right\} \mathbf{1}_{A_{n}^{m}} \mid \mathcal{F}_{n+m}\right]\right],
\end{aligned}
$$

where $\mathcal{F}_{k}=\mathcal{F}\left\{X_{0}, \ldots, X_{k}\right\}$ for any $k \geq 0$. Note that, for any $\lambda>0$,

$$
\begin{aligned}
\mathrm{E}\left[\exp \left\{-\lambda Y_{n+m+1}\right\} \mathbf{1}_{A_{n}^{m}} \mid \mathcal{F}_{n+m}\right] & =\mathbf{1}_{A_{n}^{m}} \exp \left\{-2^{-(n+m)} Y_{n+m} F\left(X_{n+m}, 2^{n+m+1} \lambda\right)\right\} \\
& \leq \mathbf{1}_{A_{n}^{m}} \exp \left\{-2^{-(n+m)} Y_{n+m} \frac{1-\left(X_{n+m} \wedge 0.5\right)}{2} 2^{n+m+1} \lambda\right\} \\
& \leq \mathbf{1}_{A_{n}^{m}} \exp \left\{-Y_{n+m}\left(1-\left(X_{n+m} \wedge 0.5\right)\right) \lambda\right\} \\
& \leq \mathbf{1}_{A_{n}^{m}} \exp \left\{-Y_{n+m}\left(1-\frac{1}{2^{(n+m) / 2}}\right) \lambda\right\} .
\end{aligned}
$$

Therefore,

$$
\mathrm{E}\left[\exp \left\{-\lambda Y_{n+m+1}\right\} \mathbf{1}_{A_{n}^{m+1}}\right] \leq \mathrm{E}\left[\mathbf{1}_{A_{n}^{m}} \exp \left\{-Y_{n+m}\left(1-\frac{1}{2^{(n+m) / 2}}\right) \lambda\right\}\right] .
$$

Recursively using (3.26), we obtain

$$
\mathrm{E}\left[\exp \left\{-\lambda Y_{n+m+1}\right\} \mathbf{1}_{A_{n}^{m}} \leq \mathrm{E}\left[\mathbf{1}_{A_{n}^{0}} \exp \left\{-\lambda Y_{n} \prod_{k=0}^{m}\left(1-\frac{1}{2^{(n+k) / 2}}\right)\right\}\right] .\right.
$$

Hence, for any $\lambda>0$ and $n \geq 2$,

$$
\begin{aligned}
\frac{p}{2} & \leq \lim _{m \rightarrow+\infty} \mathrm{E}\left[\mathbf{1}_{A_{n}^{0}} \exp \left\{-\lambda Y_{n} \prod_{k=0}^{m}\left(1-\frac{1}{2^{(n+k) / 2}}\right)\right\}\right] \\
& \leq \mathrm{E}\left[\exp \left\{-\lambda Y_{n} \prod_{k=0}^{+\infty}\left(1-\frac{1}{2^{(n+k) / 2}}\right)\right\}\right] \\
& \leq \mathrm{E}\left[\exp \left\{-\lambda Y_{n} \exp \left\{-\frac{4}{2^{n / 2}(2-\sqrt{2})}\right\}\right\}\right],
\end{aligned}
$$

where the last inequality follows from the fact that, for $n \geq 2$,

$\prod_{k=0}^{+\infty}\left(1-\frac{1}{2^{(n+k) / 2}}\right) \geq \frac{1}{\prod_{k=0}^{+\infty}\left(1+2 / 2^{(n+k) / 2}\right)} \geq \exp \left\{-\sum_{k=0}^{+\infty} \frac{2}{2^{(n+k) / 2}}\right\}=\exp \left\{-\frac{2^{-(n-4) / 2}}{2-\sqrt{2}}\right\}$.

However, letting $\lambda \rightarrow+\infty$, the right-hand side of (3.27) becomes 0 , since $Y_{n}>0$ a.s. The contradiction shows that $\mathrm{P}(Y=0)=0$. Consequently, $\mathrm{P}(0<Y<+\infty)=1$. 


\section{General speed of extinction}

The purpose of this section is to find a suitable sequence $\left\{c_{n}\right\}$ and some sufficient conditions such that $Y_{n}=X_{n} / c_{n}$ converges a.s. to a proper, nondegenerate random variable. The main tool in this section is the growth model.

We consider a general growth model $W=\left\{W_{n}, n \geq 0\right\}$ defined recursively as follows:

$$
W_{0}>0 \quad \text { a.s., } \quad W_{n+1}=\tilde{g}\left(W_{n}\right)+\zeta_{n+1}+\tilde{R}_{n+1} \quad \text { for any } n \geq 0,
$$

where $\left\{\zeta_{n}\right\},\left\{\tilde{R}_{n}\right\}$, and the function $\tilde{g}:(0,+\infty) \rightarrow(0,+\infty)$ satisfy some conditions. Küster [3] chose the following assumptions.

(H1) $\tilde{g}(w)>w$ for $w \geq w_{0}>0$.

(H2) For $w \geq w_{0}, \tilde{g}(w)$ is increasing and $\tilde{g}(w) / w$ is nonincreasing.

(H3) $\zeta_{n}$ is measurable with respect to $\tilde{\mathcal{F}}_{n} \supset \mathcal{F}\left\{W_{0}, W_{1}, \ldots, W_{n}\right\}$ and $\mathrm{E}\left[\zeta_{n} \mid \tilde{\mathcal{F}}_{n-1}\right]=0$.

(H4) There is a function $\tilde{\sigma}^{2}:(0,+\infty) \rightarrow(0,+\infty)$ such that

$$
\mathrm{E}\left[\zeta_{n+1}^{2} \mathbf{1}_{\left\{W_{n} \geq w_{0}\right\}} \mid \tilde{\mathcal{F}}_{n}\right] \leq \tilde{\sigma}^{2}\left(W_{n}\right) \mathbf{1}_{\left\{W_{n} \geq w_{0}\right\}} \text { a.s. }
$$

where

$$
\tilde{\sigma}^{2}(w)=o(1) \tilde{g}^{2}(w) \ln \left(\frac{\tilde{g}(w)}{w}\right)<\frac{\tilde{g}^{2}(w) \ln (\tilde{g}(w) / w)}{2 b^{-1}+2(1-3 b)^{-2}}
$$

for some $b \in\left(0, \frac{1}{3}\right)$ and every $w \geq w_{0}$.

(H5) Let $w_{n+1}=\tilde{g}\left(w_{n}\right)$ for any $n \geq 0$. If $\left\{y_{n}, n \geq 0\right\}$ is a sequence with

$$
\liminf _{n \rightarrow+\infty} \frac{\ln y_{n}}{\ln w_{n}} \geq 1
$$

then $\sum_{n=1}^{+\infty} \tilde{\sigma}^{2}\left(y_{n}\right) / \tilde{g}^{2}\left(y_{n}\right)<+\infty$.

Then a result was proved as follows.

Lemma 4.1. For the process $\left\{W_{n}, n \geq 0\right\}$ given by (4.1), suppose that (H1)-(H5) hold. If the random variables $\tilde{R}_{n}, n \geq 0$, satisfy

$$
\sum_{n=0}^{+\infty} \frac{\left|\tilde{R}_{n+1}\right|}{\tilde{g}\left(W_{n}\right)} \mathbf{1}_{\left\{W_{n} \geq w_{0}\right\}}<+\infty \text { a.s. }
$$

then

$$
\frac{W_{n}}{w_{n}} \rightarrow W \quad \text { a.s. with } \mathrm{P}(0<W<+\infty)=\mathrm{P}\left(\limsup _{n \rightarrow+\infty} W_{n}>w_{0}\right) .
$$

Remark 4.1. This result was stated as Proposition 1 in [3], where condition (4.2) was written as

$$
\sum_{n=0}^{+\infty} \frac{\left|\tilde{R}_{n}\right|}{\tilde{g}\left(W_{n}\right)} \mathbf{1}_{\left\{W_{n} \geq w_{0}\right\}}<+\infty \quad \text { a.s. }
$$

From the proof of Proposition 1 of [3], it is evident that expression (4.3) published in [3] includes a typo. The correct formula is (4.2). 


\subsection{An auxiliary process and some lemmas}

Now we introduce an auxiliary process $Z=\left\{Z_{n}\right\}$ with $Z_{n}=1 / X_{n}$ for any $n \geq 0$, using the conventions that $\frac{1}{0}=:+\infty$ and $1 /+\infty=: 0$. Recall that $X=\left\{X_{n}, n \geq 0\right\}$ is an extinct continuous-state PSDBP with $X_{0}>0$ and RCF

$$
F(x, \lambda)=\gamma(x) \lambda+\int_{0+}^{+\infty}\left(1-\mathrm{e}^{-\lambda s}\right) v(x, \mathrm{~d} s)
$$

where $1>\gamma(x)>0$. Then from $0<X_{n}<+\infty$ a.s. we know that $0<Z_{n}<+\infty$ a.s. Furthermore, from $X_{n} \rightarrow 0$ a.s., it follows that $Z_{n} \rightarrow+\infty$ a.s. Let

$$
\mathcal{F}_{n}:=\mathscr{F}\left(Z_{0}, Z_{1}, \ldots, Z_{n}\right)=\mathcal{F}\left(X_{0}, X_{1}, \ldots, X_{n}\right) .
$$

Define

$$
G(x):=\int_{0}^{+\infty} \exp \left\{-\frac{1}{x} F\left(x^{-1}, \lambda\right)\right\} \mathrm{d} \lambda .
$$

Then, for any $x>0$,

$$
G(x) \leq \int_{0}^{+\infty} \exp \left\{-\frac{1}{x} \gamma\left(x^{-1}\right) \lambda\right\} \mathrm{d} \lambda=\frac{x}{\gamma\left(x^{-1}\right)}<+\infty .
$$

Therefore, for any $n \geq 0$, we can calculate the conditional expectation of $Z_{n+1}$ with respect to $\mathcal{F}_{n}$ as follows:

$$
\begin{aligned}
\mathrm{E}\left[Z_{n+1} \mid \mathcal{F}_{n}\right] & =\mathrm{E}\left[Z_{n+1} \mid Z_{n}\right] \\
& =\mathrm{E}\left[\frac{1}{X_{n+1}} \mid X_{n}\right] \\
& =\mathrm{E}\left[\int_{0}^{+\infty} \exp \left\{-\lambda X_{n+1}\right\} \mathrm{d} \lambda \mid X_{n}\right] \\
& =\int_{0}^{+\infty} \mathrm{E}\left[\exp \left\{-\lambda X_{n+1}\right\} \mid X_{n}\right] \mathrm{d} \lambda \\
& =\int_{0}^{+\infty} \exp \left\{-X_{n} F\left(X_{n}, \lambda\right)\right\} \mathrm{d} \lambda \\
& =\int_{0}^{+\infty} \exp \left\{-\frac{1}{Z_{n}} F\left(Z_{n}^{-1}, \lambda\right)\right\} \mathrm{d} \lambda \\
& =G\left(Z_{n}\right) .
\end{aligned}
$$

Consequently,

$$
Z_{n+1}=G\left(Z_{n}\right)+\xi_{n+1}=g\left(Z_{n}\right)+\xi_{n+1}+R_{n+1} \quad \text { for any } n \geq 0,
$$

where $\xi_{n+1}=Z_{n+1}-G\left(Z_{n}\right), g(x)=x / \gamma\left(x^{-1}\right), R_{n+1}=G\left(Z_{n}\right)-g\left(Z_{n}\right)$, and $Z_{0}=X_{0}^{-1}$. Below, we regard $Z$ as a growth model and prove that if assumptions (A) and (B1)-(B3) hold, then conditions (H1)-(H5) are true for $Z$.

For any $x>0$, let

$$
\phi(x)=\exp \left\{-\frac{1}{x} \int_{0+}^{+\infty}\left(1-\mathrm{e}^{-s u}\right) v\left(x^{-1}, \mathrm{~d} u\right)\right\}-\exp \left\{-\frac{2}{x} \int_{0+}^{+\infty}\left(1-\mathrm{e}^{-s u}\right) v\left(x^{-1}, \mathrm{~d} u\right)\right\},
$$


and define

$$
\sigma^{2}(x):=\int_{0}^{+\infty} 4 s \exp \left\{-\frac{2}{x} \gamma\left(x^{-1}\right) s\right\} \phi(x) \mathrm{d} s .
$$

If $\int_{0+}^{+\infty} u v(x, \mathrm{~d} u)<+\infty, x>0$, then, by the inequality $1-\mathrm{e}^{-x} \leq x$,

$$
\phi(x) \leq \frac{1}{x} \int_{0+}^{+\infty}\left(1-\mathrm{e}^{-s u}\right) v\left(x^{-1}, \mathrm{~d} u\right) \leq \frac{s}{x} \int_{0+}^{+\infty} u v\left(x^{-1}, \mathrm{~d} u\right)<+\infty,
$$

and, hence,

$$
\sigma^{2}(x) \leq \int_{0}^{+\infty} 4 \frac{s}{x} \exp \left\{-\frac{2}{x} \gamma\left(x^{-1}\right) s\right\} \int_{0+}^{+\infty}\left(1-\mathrm{e}^{-s u}\right) \nu\left(x^{-1}, \mathrm{~d} u\right) \mathrm{d} s<+\infty .
$$

Therefore, assumption (B1) implies that $\sigma^{2}(x)$ is well defined for every $x>0$. We have the following lemmas.

Lemma 4.2. If assumptions ( $A)$ and (B2) hold, then

1. $g(z)>z$ for $z \geq z_{0}$, where $z_{0} \geq x_{0}^{-1}$ is a given constant;

2. for $z \geq z_{0}, g(z)$ is increasing while $g(z) / z$ is nonincreasing.

Proof. The proof is straightforward and is thus omitted.

Lemma 4.3. If assumptions (A), (B1), and (B3) hold, then

$$
\mathrm{E}\left[\xi_{n+1}^{2} \mathbf{1}_{\left\{Z_{n} \geq z_{0}\right\}} \mid \mathcal{F}_{n}\right] \leq \sigma^{2}\left(Z_{n}\right) \mathbf{1}_{\left\{Z_{n} \geq z_{0}\right\}} \text { a.s. }
$$

and

$$
\lim _{z \rightarrow+\infty} \frac{\sigma^{2}(z)}{g^{2}(z) \ln (g(z) / z)}=0 .
$$

Proof. Note that, by assumption (A),

$$
\lim _{z \rightarrow+\infty} \frac{\sigma^{2}(z)}{g^{2}(z) \ln (g(z) / z)}=\lim _{z \rightarrow+\infty} \frac{\sigma^{2}(z) \gamma\left(z^{-1}\right)}{z^{2} \ln \left(\gamma^{-1}\left(z^{-1}\right)\right)}=\frac{\gamma}{-\ln \gamma} \lim _{z \rightarrow+\infty} \frac{\sigma^{2}(z)}{z^{2}},
$$

and that, for sufficiently large $z>0$, by (4.5) and assumption (B3),

$$
\begin{aligned}
0 & \leq \frac{\sigma^{2}(z)}{z^{2}} \\
& \leq \frac{1}{z^{3}} \int_{0}^{+\infty} 4 s \exp \left\{-\frac{2}{z} \gamma\left(z^{-1}\right) s\right\} \int_{0+}^{+\infty}\left(1-\mathrm{e}^{-s u}\right) v\left(z^{-1}, \mathrm{~d} u\right) \mathrm{d} s \\
& \leq \frac{1}{z^{3}} \int_{0}^{+\infty} 4 s \exp \left\{-\frac{2}{z} \gamma\left(z^{-1}\right) s\right\} \int_{0+}^{+\infty}\left(1-\mathrm{e}^{-s u}\right) \eta(\mathrm{d} u) \mathrm{d} s \\
& =\frac{1}{z} \int_{0}^{+\infty} 4 s \exp \left\{-2 \gamma\left(z^{-1}\right) s\right\} f(z s) \mathrm{d} s,
\end{aligned}
$$

where $f(s)=\int_{0+}^{+\infty}\left(1-\mathrm{e}^{-s u}\right) \eta(\mathrm{d} u)$. Since

$$
\lim _{s \rightarrow+\infty} \frac{f(s)}{s}=0,
$$


we know that, as $z \rightarrow+\infty, f(z s) / z s \rightarrow 0$ for $s>0$. Furthermore, by (4.8), there exists an $M>0$ such that $f(s) \leq M s$, and, hence, for sufficiently large $z$,

$$
\frac{1}{z} 4 s \exp \left\{-2 \gamma\left(z^{-1}\right) s\right\} f(z s) \leq 4 s^{2} M \exp \left\{-2 \gamma\left(z^{-1}\right) s\right\} \leq 4 s^{2} M \mathrm{e}^{-s \gamma}
$$

follows from the fact that $\gamma\left(z^{-1}\right)$ tends to $\gamma>0$ as $z \rightarrow+\infty$. Applying the dominated convergence theorem to (4.7), we find that $\sigma^{2}(z) / z^{2} \rightarrow 0$ as $z \rightarrow+\infty$. Then, from (4.6), it follows that

$$
\lim _{z \rightarrow+\infty} \frac{\sigma^{2}(z)}{g^{2}(z) \ln (g(z) / z)}=0 .
$$

On the other hand, from the fact that

$$
\begin{aligned}
\mathrm{E}\left[\frac{1}{X_{n+1}^{2}} \mid X_{n}\right] & =\mathrm{E}\left[\int_{0}^{+\infty} \int_{0}^{+\infty} \exp \left\{-(s+t) X_{n+1}\right\} \mathrm{d} s \mathrm{~d} t \mid X_{n}\right] \\
& =\int_{0}^{+\infty} t \exp \left\{-X_{n} F\left(X_{n}, t\right)\right\} \mathrm{d} t
\end{aligned}
$$

and the convexity of $F$, it follows that

$$
\begin{aligned}
\mathrm{E}\left[\xi_{n+1}^{2} \mid \mathcal{F}_{n}\right]= & \int_{0}^{+\infty} t \exp \left\{-Z_{n}^{-1} F\left(Z_{n}^{-1}, t\right)\right\} \mathrm{d} t \\
& -\int_{0}^{+\infty} \exp \left\{-Z_{n}^{-1} F\left(Z_{n}^{-1}, s\right)\right\} \mathrm{d} s \int_{0}^{+\infty} \exp \left\{-Z_{n}^{-1} F\left(Z_{n}^{-1}, u\right)\right\} \mathrm{d} u \\
\leq & \int_{0}^{+\infty} s \exp \left\{-Z_{n}^{-1} F\left(Z_{n}^{-1}, s\right)\right\} \mathrm{d} s \\
& -\int_{0}^{+\infty} \int_{0}^{+\infty} \exp \left\{-2 Z_{n}^{-1} F\left(Z_{n}^{-1}, \frac{s+u}{2}\right)\right\} \mathrm{d} s \mathrm{~d} u \\
\leq & \int_{0}^{+\infty} 4 s \exp \left\{-Z_{n}^{-1} F\left(Z_{n}^{-1}, 2 s\right)\right\} \mathrm{d} s-\int_{0}^{+\infty} 4 s \exp \left\{-2 Z_{n}^{-1} F\left(Z_{n}^{-1}, s\right)\right\} \mathrm{d} s \\
= & \int_{0}^{+\infty} 4 s \exp \left\{-2 Z_{n}^{-1} F\left(Z_{n}^{-1}, s\right)\right\} \\
& \quad \times\left(\exp \left\{2 Z_{n}^{-1} F\left(Z_{n}^{-1}, s\right)-Z_{n}^{-1} F\left(Z_{n}^{-1}, 2 s\right)\right\}-1\right) \mathrm{d} s .
\end{aligned}
$$

Then, by the fact that, for any $x>0$,

$$
2 F(x, s)-F(x, 2 s)=\int_{0+}^{+\infty}\left(1-\mathrm{e}^{-s u}\right)^{2} v(x, \mathrm{~d} u) \leq \int_{0+}^{+\infty}\left(1-\mathrm{e}^{-s u}\right) v(x, \mathrm{~d} u),
$$

we obtain

$$
\begin{aligned}
\mathrm{E}\left[\xi_{n+1}^{2} \mid \mathcal{F}_{n}\right] \leq \int_{0}^{+\infty} & 4 s \exp \left\{-2 Z_{n}^{-1} F\left(Z_{n}^{-1}, s\right)\right\} \\
& \quad \times\left(\exp \left\{Z_{n}^{-1} \int_{0+}^{+\infty}\left(1-\mathrm{e}^{-s u}\right) v\left(Z_{n}^{-1}, \mathrm{~d} u\right)\right\}-1\right) \mathrm{d} s \\
& =\int_{0}^{+\infty} 4 s \exp \left\{-2 Z_{n}^{-1} \gamma\left(Z_{n}^{-1}\right) s\right\} \phi\left(Z_{n}\right) \mathrm{d} s \\
= & \sigma^{2}\left(Z_{n}\right) .
\end{aligned}
$$

This completes the proof. 
Lemma 4.4. Suppose that assumptions (A) and (B1)-(B3) hold. Let $z_{n+1}=g\left(z_{n}\right)$ for any $n \geq 0$. If, for any $n \geq 0, y_{n}>0$ and $\liminf _{n \rightarrow+\infty} \ln y_{n} / \ln z_{n} \geq 1$, then

$$
\sum_{n=1}^{+\infty} \frac{\sigma^{2}\left(y_{n}\right)}{g^{2}\left(y_{n}\right)}<+\infty
$$

Proof. By assumptions (A) and (B2), we know that

$$
\gamma\left(x_{0}^{-1}\right) \leq \gamma_{0}:=\gamma\left(z_{0}\right) \leq \gamma\left(z^{-1}\right) \leq \gamma \quad \text { for any } z \geq z_{0} .
$$

Hence, it is easy to obtain $z_{n} \geq z_{0} / \gamma^{n}$. Then $\liminf _{n \rightarrow+\infty} \ln y_{n} / \ln z_{n} \geq 1$ indicates that there exist some $k>0$ and $\beta>1$ such that, for all $n \geq k, z_{0} / \gamma^{n} \geq \beta^{n}$ and $y_{n} \geq \beta^{n} \geq z_{0}$. Consequently,

$$
\sum_{n=0}^{+\infty} \frac{\sigma^{2}\left(y_{n}\right)}{g^{2}\left(y_{n}\right)}=\sum_{n=0}^{+\infty} \frac{\gamma^{2}\left(y_{n}^{-1}\right) \sigma^{2}\left(y_{n}\right)}{y_{n}^{2}} \leq \sum_{n=0}^{+\infty} \frac{\sigma^{2}\left(y_{n}\right)}{y_{n}^{2}}=\sum_{n=0}^{k} \frac{\sigma^{2}\left(y_{n}\right)}{y_{n}^{2}}+\sum_{n=k+1}^{+\infty} \frac{\sigma^{2}\left(y_{n}\right)}{y_{n}^{2}}
$$

Using assumption (B3) for the case $y_{n} \geq z_{0}$, from (4.5) we further obtain

$$
\begin{aligned}
\sum_{n=0}^{+\infty} \frac{\sigma^{2}\left(y_{n}\right)}{g^{2}\left(y_{n}\right)} & \leq \sum_{n=0}^{k} \frac{\sigma^{2}\left(y_{n}\right)}{y_{n}^{2}}+\sum_{n=k+1}^{+\infty} \frac{1}{y_{n}} \int_{0}^{+\infty} 4 s \exp \left\{-2 \gamma\left(y_{n}^{-1}\right) s\right\} f\left(y_{n} s\right) \mathrm{d} s \\
& =\sum_{n=0}^{k} \frac{\sigma^{2}\left(y_{n}\right)}{y_{n}^{2}}+\int_{0}^{+\infty} \sum_{n=k+1}^{+\infty} \frac{1}{y_{n}} 4 s \exp \left\{-2 \gamma\left(y_{n}^{-1}\right) s\right\} f\left(y_{n} s\right) \mathrm{d} s \\
& \leq \sum_{n=0}^{k} \frac{\sigma^{2}\left(y_{n}\right)}{y_{n}^{2}}+\int_{0}^{+\infty} 4 s \exp \left\{-2 \gamma_{0} s\right\} \sum_{n=k+1}^{+\infty} \frac{1}{\beta^{n}} f\left(\beta^{n} s\right) \mathrm{d} s,
\end{aligned}
$$

where we have used the fact that $f(x s) / x$ is nonincreasing with respect to $x$. Note that

$$
\begin{aligned}
\sum_{n=0}^{+\infty} \frac{1}{\beta^{n}} f\left(\beta^{n} s\right) & \leq \int_{0}^{+\infty} \frac{1}{\beta^{x}} f\left(\beta^{x} s\right) \mathrm{d} x \\
& =\int_{0}^{1} f\left(\frac{s}{y}\right) \frac{1}{\ln \beta} \mathrm{d} y \\
& =\frac{1}{\ln \beta} \int_{0+}^{+\infty}\left(1-\int_{u}^{+\infty} \mathrm{e}^{-s y} \frac{u}{y^{2}} \mathrm{~d} y\right) \eta(\mathrm{d} u) \\
& =\frac{1}{\ln \beta} \int_{0+}^{+\infty}\left(1-\mathrm{e}^{-s u}+u \int_{u}^{+\infty} \frac{s}{y} \mathrm{e}^{-s y} \mathrm{~d} y\right) \eta(\mathrm{d} u) \\
& \leq \frac{1}{\ln \beta} \int_{0+}^{+\infty}\left(1-\mathrm{e}^{-s u}+u \int_{u}^{+\infty} \frac{s}{y} \frac{1}{1+s y} \mathrm{~d} y\right) \eta(\mathrm{d} u) \\
& =\frac{1}{\ln \beta} \int_{0+}^{+\infty}\left(1-\mathrm{e}^{-s u}+u s(\ln (1+s u)-\ln (u))\right) \eta(\mathrm{d} u)
\end{aligned}
$$

Since

$$
\ln (1+s u)-\ln (u) \leq \begin{cases}\ln (1+s)-\ln (u), & 0<u \leq 1 \\ \ln (1+s), & u>1\end{cases}
$$


we obtain

$$
1-\mathrm{e}^{-s u}+u s(\ln (1+s u)-\ln (u)) \leq s u(1+s)-s u \ln (u) \mathbf{1}_{\{u \leq 1\}} .
$$

Assumption (B3) indicates that there exists a positive constant $M$ satisfying

$$
\int_{0+}^{+\infty} u \eta(\mathrm{d} u)+\int_{0+}^{1} u|\ln u| \eta(\mathrm{d} u)<M .
$$

Hence, using (4.13), we obtain

$$
\int_{0+}^{+\infty}\left(1-\mathrm{e}^{-s u}+u s(\ln (1+s u)-\ln (u))\right) \eta(\mathrm{d} u)<M\left(s^{2}+2 s\right) .
$$

Combining (4.10), (4.11), and (4.14), we immediately obtain the desired conclusion.

Lemma 4.5. Suppose that assumptions (A) and (B1)-(B3) hold. The random variables $\left\{R_{n}\right\}_{n \geq 0}$ satisfy

$$
\sum_{n=0}^{+\infty} \frac{\left|R_{n+1}\right|}{g\left(Z_{n}\right)} \mathbf{1}_{\left\{Z_{n} \geq z_{0}\right\}}<+\infty \text { a.s. }
$$

Proof. Using integration by parts, we obtain

$$
G(x)=\frac{x}{\gamma\left(x^{-1}\right)}-\int_{0+}^{+\infty} \frac{s}{\gamma\left(x^{-1}\right)} \int_{0}^{+\infty} \exp \left\{-x^{-1} F\left(x^{-1}, \lambda\right)+\lambda s\right\} \nu\left(x^{-1}, \mathrm{~d} s\right) \mathrm{d} \lambda .
$$

Since $\gamma(x) \lambda \leq F(x, \lambda)$ for all $x>0$,

$$
G(x) \geq \frac{x}{\gamma\left(x^{-1}\right)}-\int_{0+}^{+\infty} \frac{1}{\gamma\left(x^{-1}\right)} \frac{s x}{s x+\gamma\left(x^{-1}\right)} v\left(x^{-1}, \mathrm{~d} s\right) .
$$

Hence,

$$
\left|R_{n+1}\right|=\left|G\left(Z_{n}\right)-g\left(Z_{n}\right)\right| \leq \int_{0+}^{+\infty} \frac{1}{\gamma\left(Z_{n}^{-1}\right)} \frac{s Z_{n}}{s Z_{n}+\gamma\left(Z_{n}^{-1}\right)} v\left(Z_{n}^{-1}, \mathrm{~d} s\right)
$$

and

$$
\frac{\left|R_{n+1}\right|}{g\left(Z_{n}\right)} \mathbf{1}_{\left\{Z_{n} \geq z_{0}\right\}} \leq \int_{0+}^{+\infty} \frac{1}{\gamma\left(Z_{n}^{-1}\right)} \frac{s \gamma\left(Z_{n}^{-1}\right)}{s Z_{n}+\gamma\left(Z_{n}^{-1}\right)} v\left(Z_{n}^{-1}, \mathrm{~d} s\right) \mathbf{1}_{\left\{Z_{n} \geq z_{0}\right\}} .
$$

Let

$$
A_{k}=\bigcap_{n=k}^{+\infty}\left\{Z_{n} \geq \frac{1}{m^{n / 2}}\right\}, \quad k=1,2, \ldots
$$

Since $m(x) \rightarrow m<1$ as $x \rightarrow 0$ and assumption (A) holds, by Corollary 3.1 we find that $X_{n} / m^{n / 2} \rightarrow 0$ a.s., which indicates that

$$
\lim _{k \rightarrow \infty} \mathrm{P}\left(A_{k}\right)=1
$$

From (4.9), it follows that

$$
\frac{1}{\gamma\left(Z_{n}^{-1}\right)} \frac{s \gamma\left(Z_{n}^{-1}\right)}{s Z_{n}+\gamma\left(Z_{n}^{-1}\right)} \mathbf{1}_{\left\{Z_{n} \geq z_{0}\right\}} \leq \frac{1}{\gamma_{0}} \frac{s \gamma}{s Z_{n}+\gamma} \mathbf{1}_{\left\{Z_{n} \geq z_{0}\right\}} .
$$


Then, for any $k \geq 1$, using (4.16) and (4.18), we obtain

$$
\begin{aligned}
\mathbf{1}_{A_{k}} \sum_{n=0}^{+\infty} \frac{\left|R_{n+1}\right|}{g\left(Z_{n}\right)} \mathbf{1}_{\left\{Z_{n} \geq z_{0}\right\}} \leq & \sum_{n=0}^{+\infty} \int_{0+}^{+\infty} \frac{1}{\gamma_{0}} \frac{s \gamma}{s Z_{n}+\gamma} v\left(Z_{n}^{-1}, \mathrm{~d} s\right) \mathbf{1}_{\left\{Z_{n} \geq z_{0}\right\}} \mathbf{1}_{A_{k}} \\
\leq & \sum_{n=0}^{k-1} \int_{0+}^{+\infty} \frac{\gamma s}{\gamma_{0}\left(s Z_{n}+\gamma\right)} \eta(\mathrm{d} s) \\
& +\int_{0+}^{+\infty} \frac{1}{\gamma_{0}} \sum_{n=k}^{+\infty} \frac{s \gamma}{s m^{-n / 2}+\gamma} \eta(\mathrm{d} s) .
\end{aligned}
$$

Assumption (B3) implies that

$$
\begin{aligned}
\int_{0+}^{+\infty} \frac{1}{\gamma_{0}} \sum_{n=k}^{+\infty} \frac{s \gamma}{s m^{-n / 2}+\gamma} \eta(\mathrm{d} s) & \leq \int_{0+}^{+\infty} \frac{1}{\gamma_{0}} \int_{0}^{+\infty} \frac{s \gamma}{s m^{-x / 2}+\gamma} \mathrm{d} x \eta(\mathrm{d} s) \\
& \leq \int_{0+}^{+\infty} \frac{1}{\gamma_{0}} \int_{0}^{1} \frac{2 s \gamma}{s+\gamma y} \frac{\mathrm{d} y}{|\ln m|} \eta(\mathrm{d} s) \\
& =\frac{2}{\gamma_{0}|\ln m|} \int_{0+}^{+\infty} s(\ln (s+\gamma)-\ln s) \eta(\mathrm{d} s) \\
& \leq \frac{2}{\gamma_{0}|\ln m|}\left(\int_{0+}^{+\infty} s \ln (1+\gamma) \eta(\mathrm{d} s)-\int_{0+}^{1} s \ln (s) \eta(\mathrm{d} s)\right) \\
& <+\infty
\end{aligned}
$$

and that

$$
\sum_{n=0}^{k-1} \int_{0+}^{+\infty} \frac{\gamma s}{\gamma_{0}\left(s Z_{n}+\gamma\right)} \eta(\mathrm{d} s) \leq \frac{k}{\gamma_{0}} \int_{0+}^{+\infty} s \eta(\mathrm{d} s)<+\infty .
$$

From (4.19)-(4.21), it follows that

$$
\mathbf{1}_{A_{k}} \sum_{n=0}^{+\infty}\left|R_{n+1}\right| g\left(Z_{n}\right)^{-1} \mathbf{1}_{\left\{Z_{n} \geq z_{0}\right\}}<+\infty \quad \text { for any } k \geq 1 .
$$

Then (4.17) implies that

$$
\sum_{n=0}^{+\infty}\left|R_{n+1}\right| g\left(Z_{n}\right)^{-1} \mathbf{1}_{\left\{Z_{n} \geq z_{0}\right\}}<+\infty \quad \text { a.s. }
$$

This completes the proof.

\subsection{Proper and nondegenerate limits}

Now we give the proof of Theorem 2.3.

Proof of Theorem 2.3. Obviously, $\xi_{n}$ is measurable with respect to $\mathscr{F}_{n}$ and $\mathrm{E}\left[\xi_{n} \mid \mathcal{F}_{n-1}\right]=0$. In addition, from Lemma 4.3 we know that there exists a $\tilde{z}_{0} \geq x_{0}^{-1}$ such that

$$
\sigma^{2}(z)<\frac{g^{2}(z) \ln (g(z) / z)}{2 b^{-1}+2(1-3 b)^{-2}}
$$

for some $b \in\left(0, \frac{1}{3}\right)$ and every $z \geq \tilde{z}_{0}$. Without loss of generality, we rewrite $\tilde{z}_{0}$ as $z_{0}$. Then, 
using Lemmas 4.2-4.5, we can show that the conditions of Lemma 4.1 hold for $Z=\left\{Z_{n}\right\}$ and $\left\{z_{n}\right\}$. Applying Lemma 4.1, we find that $Z_{n} / z_{n}$ converges a.s. to a proper, nondegenerate random variable $L \in(0,+\infty)$. Let $c_{n}=z_{n}^{-1}$. Since $z_{n} \rightarrow+\infty$, we have

$$
\lim _{n \rightarrow+\infty} \frac{c_{n+1}}{c_{n}}=\lim _{n \rightarrow+\infty} \frac{z_{n}}{z_{n+1}}=\lim _{n \rightarrow+\infty} \gamma\left(z_{n}^{-1}\right)=\gamma
$$

Moreover,

$$
\lim _{n \rightarrow+\infty} \frac{X_{n}}{c_{n}}=\lim _{n \rightarrow+\infty} \frac{z_{n}}{Z_{n}}=\frac{1}{L} \in(0,+\infty) \text { a.s. }
$$

This completes the proof.

Remark 4.2. Under assumptions (A) and (B1)-(B3), Theorem 2.3 shows that $X_{n} / c^{n} \rightarrow 0$ a.s. for $c>\gamma$ and $X_{n} / c^{n} \rightarrow+\infty$ a.s. for $c<\gamma$.

Furthermore, we have the following corollary.

Corollary 4.1. Suppose that assumptions (A), (B1), and (B3) hold. Let $\gamma(x) \equiv \gamma$ for sufficiently small $x$. Then we can take $c_{n}=c_{0} \gamma^{n}$ for some $c_{0}>0$ and all $n \geq 0$ such that $X_{n} / c_{n}$ converges a.s. to a proper, nondegenerate random variable. Therefore, $X_{n} / \gamma^{n}$ converges a.s. to a proper, nondegenerate random variable.

Proof. Since $\gamma(x) \equiv \gamma$ for sufficiently small $x$, there exists an $x_{0}>0$ such that, for all $x \in\left(0, x_{0}\right), \gamma(x) \equiv \gamma$, which implies that assumption (B2) holds. Then by Theorem 2.3 we can have $\left\{c_{n}\right\}$ such that $X_{n} / c_{n}$ converges a.s. to a proper, nondegenerate random variable, where $c_{n}^{-1}=z_{n}=g\left(z_{n-1}\right)$ for any $n \geq 1, g(x)=x / \gamma\left(x^{-1}\right)$, and $z_{0}$ is any sufficiently large constant. Note that, when $z_{0}>x_{0}^{-1}, z_{n}=z_{0} / \gamma^{n}$, and, hence, $c_{n}=c_{0} \gamma^{n}$, where $c_{0}=z_{0}^{-1}>0$.

By Corollary 4.1 we can obtain a group of sufficient conditions such that the speed of extinction is $\gamma^{n}$. However, Example 2.2 is not included in this corollary. For an application of Theorem 2.3 to Jiřina processes, see Corollary 2.1 .

To end, we point out that the parameter $1 / \gamma$ is the limit of the unit conditional mean of $Z_{n+1}$ with respect to $Z_{n}=z$ as $z \rightarrow+\infty$, namely,

$$
\frac{1}{\gamma}=\lim _{z \rightarrow+\infty} \frac{\mathrm{E}\left[Z_{n+1} \mid Z_{n}=z\right]}{z}=\lim _{z \rightarrow+\infty} \frac{G(z)}{z} .
$$

In fact, from (4.4), (4.15), and assumption (B3), we have

$$
\begin{aligned}
\frac{1}{\gamma} & \geq \limsup _{z \rightarrow+\infty} \frac{G(z)}{z} \\
& \geq \liminf _{z \rightarrow+\infty} \frac{G(z)}{z} \\
& \geq \frac{1}{\gamma}-\lim _{z \rightarrow+\infty} \frac{1}{z} \int_{0+}^{+\infty} \frac{s z}{s z+\gamma\left(z^{-1}\right)} v\left(\frac{1}{z}, \mathrm{~d} s\right) \\
& \geq \frac{1}{\gamma}-\lim _{z \rightarrow+\infty} \int_{0+}^{+\infty} \frac{s}{s z+\gamma_{0}} v\left(\frac{1}{z}, \mathrm{~d} s\right) \\
& \geq \frac{1}{\gamma}-\lim _{z \rightarrow+\infty} \int_{0+}^{+\infty} \frac{s}{s z+\gamma_{0}} \eta(\mathrm{d} s) \\
& =\frac{1}{\gamma} .
\end{aligned}
$$


Applying this fact to the Jiřina process, we discover that Theorem 2.1 of [8] for the subcritical case and Theorem 3.1 of [8] for the supercritical case are consistent to some extent. The two theorems seem different; the former depends on the drift parameter $\gamma$ and the latter depends on the offspring mean $m$. However, in essence, they both depend on the so-called limit of the unit conditional mean. Here $1 / \gamma$ is the limit of the unit conditional mean of $Z_{n+1}=1 / X_{n+1}$ as $Z_{n}=z \rightarrow+\infty$, and $m$ is the limit of the unit conditional mean of $X_{n+1}$ as $X_{n}=x \rightarrow+\infty$.

\section{Acknowledgement}

The author is grateful to the anonymous referee whose careful reading and several suggestions helped to improve the paper.

\section{References}

[1] JiřınA, M. (1960). Stochastic branching processes with continuous state space. Czech. Math. J. 83, $292-313$.

[2] JIŘInA, M. (1966). Asymptotic behaviour of measure-valued branching processes. Rozpr. Česk. Akad. Věd. Řada Mat. Přir. Věd. 76, 1-55.

[3] Kuster, P. (1985). Asymptotic growth of controlled Gaton-Watson processes. Ann. Prob. 13, 1157-1178.

[4] LI, Y. (2006). On a continuous-state population-size-dependent branching process and its extinction. J. Appl. Prob. 43, 195-207.

[5] LI, Y. (2009). Approximating nonlinear models of interest rates with branching processes. Acta Math. Sci., 29, 1-9 (in Chinese).

[6] LI, Y. (2009). A weak limit theorem for generalized Jiřina processes. J. Appl. Prob. 46, 453-462.

[7] LI, Y. (2009). Limit theorems for generalized Jiřina processes. Statist. Prob. Lett.. 79, 158-164.

[8] Seneta, E. And Vere-Jones, D. (1969). On a problem of M. Jiřina concerning continuous state branching processes. Czech. Math. J. 9, 277-283. 